\title{
Non-propagating Surface Modes in Dual Singly Negative Material Layers
}

\author{
Kyoung-Youm Kim* \\ Department of Optical Engineering, Sejong University, Gunja-dong, Gwangjin-gu, \\ Seoul 143-747, Korea \\ Saehwa Kim \\ Information Communications Engineering Major, Hankuk University of Foreign Studies, \\ Yongin 449-791, Korea
}

(Received August 5, 2009 : revised September 14, 2009 : accepted September 15, 2009)

\begin{abstract}
We investigate the characteristics of the surface mode formed at the single interface between dual singly negative medium layers. It is shown that the interface can support a mode which has polarization degeneracy and a vanishing propagation constant. That is, this surface mode is a polarization-independent one that does not propagate at all through the interface. The corresponding electric and magnetic field distributions become static along the longitudinal (guiding) direction (but not along the transverse direction) resulting in the formation of transverse-electric/longitudinalmagnetic or transverse-magnetic/longitudinal-electric modes.
\end{abstract}

Keywords: Metamaterials, Waveguides, Surface waves

OCIS codes : (160.3918) Metamaterials; (230.7390) Waveguides, planar; (240.6690) Surface waves; (260.2110) Electromagnetic optics

\section{INTRODUCTION}

Metamaterials, subwavelength-scale periodic metaldielectric composites have opened us a new land of materials for optics $[1,2]$. They can have doubly negative features, i.e., both negative permittivity $(\varepsilon)$ and negative permeability $(\mu)$. In these, so-called doubly negative media, light waves can propagate just like in the dielectric layers except that the phase velocity or the direction of wavevector becomes anti-parallel to the energy/power flow direction [1, 2]. There is another kind of metamaterials, called singly negative media which have either negative $\mathcal{E}$ but positive $\mu$ ( $\mathcal{E}$-negative material; note that in some cases, the $\varepsilon$-negative medium can include metal in the visible regime, which means that $\mathcal{\varepsilon}$-negative property does not always require the use of metamaterials) or positive $\mathcal{\varepsilon}$ but negative $\mu$ ( $\mu$-negative medium). Contrary to the doubly negative materials, incident light waves cannot propagate through these singly negative materials since the singly negative property, $\mathcal{E} \cdot \mu<0$, makes the longitudinal wavenumbers purely imaginary. In this sense, we can say that the singly negative media are opaque.

\footnotetext{
*Corresponding author: kykim@sejong.ac.kr
}

Recently, one of the present authors proposed the use of dual singly negative materials $[3,4]$. They include $\mathcal{E}$-negative (having relative permittivity $\mathcal{E}_{e}=-\left|\mathcal{E}_{e}\right|$ and relative permeability $\left.\mu_{e}=\left|\mu_{e}\right|\right)$ and $\mu$-negative $\left(\varepsilon_{m}=\left|\varepsilon_{m}\right|\right.$, $\left.\mu_{m}=-\left|\mu_{m}\right|\right)$ media whose impedances are matched to each other, i.e., $\sqrt{\mu_{e} / \varepsilon_{e}}=\sqrt{\mu_{m} / \varepsilon_{m}}$ or $\varepsilon_{e} / \varepsilon_{m}=\mu_{e} / \mu_{m}=\zeta_{e m}$, but not perfectly, i.e., excluding the case of $\mathcal{E}_{e}=-\varepsilon_{m}$ and $\mu_{e}=-\mu_{m}$. In previous studies, we have investigated the possible applications of such dual media, e.g., the transmission filter [3] or the polarization beam splitter [4]. Our attention in this work will be focused on (1) whether the single interface between these media can support any guiding modes which propagate like the surface plasmon polariton (SPP) waves at the metaldielectric interfaces and (2) if any, what their unique properties are.

\section{GUIDING MODE THROUTH THE INTERFACE BETWEEN DUAL SINGLY NEGATIVE MATERIALS}

\section{Solution of Maxwell's Equations}

Let us consider a single interface between the dual singly negative media (see Fig. 1). We will suppose a 
surface mode $\psi$ which propagates along this interface, say $z$ direction. $E_{y}$ (TE case) or $H_{y}$ (TM case) field corresponds to $\psi$, which can be written as

$$
\psi= \begin{cases}\hat{y} A \exp \left(\kappa_{e} x\right) \exp (j \beta z-j \omega t), & x<0 \\ \hat{y} B \exp \left(-\kappa_{m} x\right) \exp (j \beta z-j \omega t), & x>0\end{cases}
$$

where $\boldsymbol{\kappa}_{e}=\left(\beta^{2}-\mathcal{\varepsilon}_{e} \mu_{e} k_{0}^{2}\right)^{1 / 2}$ and $\boldsymbol{\kappa}_{m}=\left(\beta^{2}-\mathcal{E}_{m} \mu_{m} k_{0}^{2}\right)^{1 / 2} . k_{0}$ is the wavenumber of light in vacuum. Using the continuity conditions of $\psi$ and $(1 / \sigma)(\partial \psi / \partial x)$ at the interface (where $\sigma$ denotes $\mu$ and $\mathcal{E}$ for TE- and TM-polarized light, respectively), we can get

$$
\begin{aligned}
& A=B, \\
& \frac{\kappa_{e}}{\kappa_{m}}=-\frac{\sigma_{e}}{\sigma_{m}}\left(\equiv-\sigma_{e m}\right) .
\end{aligned}
$$

From Eq. (3), we can obtain the propagation constant of $\psi$ as

$$
\beta^{2}=k_{0}^{2} \frac{\sigma_{e m}^{2} \varepsilon_{m} \mu_{m}-\varepsilon_{e} \mu_{e}}{1-\sigma_{e m}^{2}} .
$$

Up to this point, we have followed the general formulation. Here, we apply the specific feature of the dual singly negative media: $\sigma_{e m}=\varepsilon_{e} / \mathcal{E}_{m}=\mu_{e} / \mu_{m}=\zeta_{e m}$. It leads to

$$
\beta_{T E}^{2}=k_{0}^{2} \mu_{e} \mu_{m} \frac{\varepsilon_{m} \mu_{e}-\varepsilon_{e} \mu_{m}}{\mu_{m}^{2}-\mu_{e}^{2}}=0
$$

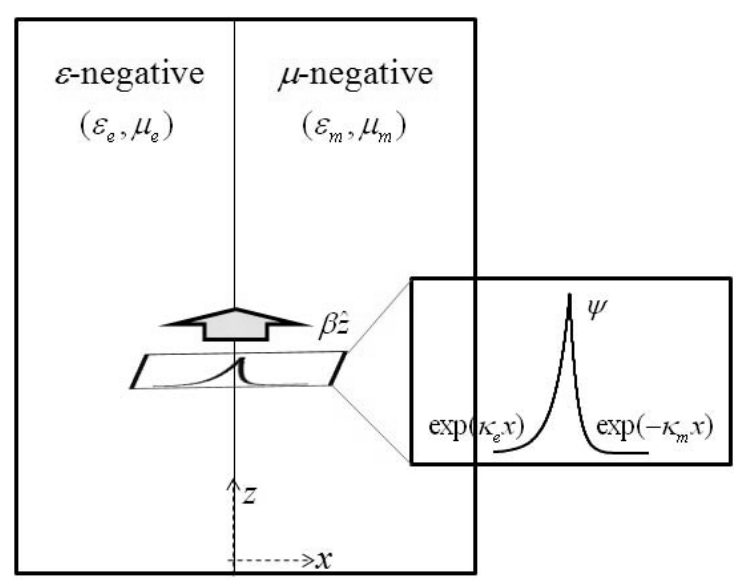

FIG. 1. A single interface between the dual singly negative media. A surface mode $\psi$ is assumed to propagate along the $z$ direction with a propagation constant of $\beta$ and decay constants of $\kappa_{e}$ and $\kappa_{m}$ in the $\varepsilon$-negative and $\mu$-negative media, respectively.

$$
\beta_{T M}^{2}=k_{0}^{2} \varepsilon_{e} \varepsilon_{m} \frac{\varepsilon_{e} \mu_{m}-\varepsilon_{m} \mu_{e}}{\varepsilon_{m}^{2}-\varepsilon_{e}^{2}}=0
$$

Then, we have $\kappa_{e}=k_{0} \sqrt{-\varepsilon_{e} \mu_{e}}$ and $\boldsymbol{\kappa}_{m}=k_{0} \sqrt{-\varepsilon_{m} \mu_{m}}$ (please recall that $\varepsilon_{e} \mu_{e}<0$ and $\varepsilon_{m} \mu_{m}<0$ ), and $\psi$ can be written as (omitting the normalization constants for brevity)

$$
\psi=\left\{\begin{array}{c}
\hat{y} \exp \left(k_{0} \sqrt{\left|\varepsilon_{e} \mu_{e}\right|} x\right) \exp (-j \omega t), \quad x<0 \\
\hat{y} \exp \left(-k_{0} \sqrt{\left|\varepsilon_{m} \mu_{m}\right|} x\right) \exp (-j \omega t), \quad x>0
\end{array}\right.
$$

We can easily see that Eq. (7) satisfies the boundary condition, Eq. (3), since we have (note that $\zeta_{e m}$ and $\sigma_{e m}$ are negative)

$$
\frac{\kappa_{e}}{\kappa_{m}}=\sqrt{\frac{-\varepsilon_{e} \mu_{e}}{-\varepsilon_{m} \mu_{m}}}=\sqrt{\left(-\zeta_{e m}\right) \cdot\left(-\zeta_{e m}\right)}=-\zeta_{e m}=-\sigma_{e m} .
$$

Therefore, we can draw a conclusion that there exists a surface mode at the single interface between dual singly negative medium layers. It has the unique features of (1) polarization degeneracy, i.e., this surface mode remains the eigenmode of the waveguide for all changes in the polarization state of light (since $\beta_{T E}=\beta_{T M}$ ), and (2) the vanishing propagation constant, i.e., $\beta=0$. That is, the surface mode is a polarization-independent one that does not propagate at all through the interface. This eigenmode field has decay constants of $\kappa_{e}=k_{0} \sqrt{\left|\varepsilon_{e} \mu_{e}\right|}$ and $\kappa_{m}=k_{0} \sqrt{\left|\varepsilon_{m} \mu_{m}\right|}$ in the $\mathcal{\varepsilon}$-negative and $\mu$-negative layers, respectively. In the following subsections, we will briefly summarize the electric and magnetic field distributions corresponding to this special mode for TE- and TM-polarization cases.

\section{TE-polarization mode}

Using Eq. (7), we have

$$
\mathbf{E}=\left\{\begin{array}{c}
\hat{y} \exp \left(k_{0} \sqrt{\left|\varepsilon_{e} \mu_{e}\right|} x\right) \exp (-j \omega t), \quad x<0 \\
\hat{y} \exp \left(-k_{0} \sqrt{\left|\varepsilon_{m} \mu_{m}\right|} x\right) \exp (-j \omega t), \quad x>0
\end{array}\right.
$$

From $\mathbf{H}=(1 / j w \mu) \nabla \times \mathbf{E}$, we can calculate the magnetic field as

$$
\mathbf{H}=\left\{\begin{array}{c}
\hat{z} \frac{1}{\eta_{e}} \exp \left(k_{0} \sqrt{\left|\varepsilon_{e} \mu_{e}\right|} x\right) \exp (-j \omega t), \quad x<0 \\
-\hat{z} \frac{1}{\eta_{m}} \exp \left(-k_{0} \sqrt{\left|\varepsilon_{m} \mu_{m}\right|} x\right) \exp (-j \omega t), \quad x>0
\end{array}\right.
$$

where $\eta_{e}=j \eta_{0} \sqrt{\left|\mu_{e} / \varepsilon_{e}\right|}$ and $\eta_{m}=j \eta_{0} \sqrt{\left|\mu_{m} / \varepsilon_{m}\right|}$ are the characteristic impedance of $\varepsilon$-negative and $\mu$-negative media, respectively ( $\eta_{0}$ denotes that of vacuum), and will be denoted as $\eta_{e}=\eta_{m}=j\left|\eta_{d}\right|$. We can see that there is no 
transverse ( $x$-directional) component of the magnetic field. Using Eqs. (9) and (10), we can obtain the following expression for the Poynting vector

$$
\mathbf{S}=\frac{1}{2} \mathbf{E} \times \mathbf{H}^{*}=\left\{\begin{array}{cc}
j \hat{x} \frac{1}{2\left|\eta_{d}\right|} \exp \left(2 k_{0} \sqrt{\varepsilon_{e} \mu_{e} \mid} x\right), \quad x<0 \\
-j \hat{x} \frac{1}{2\left|\eta_{d}\right|} \exp \left(-2 k_{0} \sqrt{\left|\varepsilon_{m} \mu_{m}\right|} x\right), \quad x>0
\end{array}\right.
$$

\section{TM-polarization mode}

For TM case, we have similarlys

$$
\mathbf{H}=\left\{\begin{array}{c}
\hat{y} \exp \left(k_{0} \sqrt{\mid \varepsilon_{e} \mu_{e}} x\right) \exp (-j \omega t), \quad x<0 \\
\hat{y} \exp \left(-k_{0} \sqrt{\left|\varepsilon_{m} \mu_{m}\right|} x\right) \exp (-j \omega t), \quad x>0
\end{array}\right.
$$

From $\mathbf{E}=-(1 / j \omega \varepsilon) \nabla \times \mathbf{H}$, we can obtain the following expressions for the electric field and the Poynting vector

$$
\begin{aligned}
& \mathbf{E}= \begin{cases}-j \hat{z}\left|\eta_{d}\right| \exp \left(k_{0} \sqrt{\left|\varepsilon_{e} \mu_{e}\right|} x\right) \exp (-j \omega t), & x<0 \\
j \hat{z}\left|\eta_{d}\right| \exp \left(-k_{0} \sqrt{\mid \varepsilon_{m} \mu_{m}} \mid x\right) \exp (-j \omega t), & x>0\end{cases} \\
& \mathbf{S}=\left\{\begin{array}{cc}
j \hat{x} \frac{\left|\eta_{d}\right|}{2} \exp \left(2 k_{0} \sqrt{\left|\varepsilon_{e} \mu_{e}\right|} x\right), & x<0 \\
-j \hat{x} \frac{\eta_{d} \mid}{2} \exp \left(-2 k_{0} \sqrt{\left|\varepsilon_{m} \mu_{m}\right|} x\right), & x>0
\end{array}\right.
\end{aligned}
$$

Similarly to the TE case, we cannot find any transverse ( $x$-directional) component of the electric field.

\section{Properties of the guiding modes}

From the above calculation and discussions, we can find the following characteristics of the mode that the single interface between the dual singly negative media supports:

(1) Both the electric and magnetic field components have no $z$-coordinate dependency. This means that all fields are constant along the $z$ direction (but not along the $x$ direction). That is, the light fields become static along the longitudinal direction. These characteristics remind us of similar characteristics found in the zero-refractive-index or $\mathcal{E}$-zero media [5-7] where the light fields become static along all directions. However, in our structure, although the light fields do not have any spatial phase variation along all directions, they have spatial amplitude variation along the transverse direction. This is the point where our structure has unique features.

(2) As a result of above property, there is no transverse magnetic field for the TE light mode: it involves only the longitudinal magnetic field, i.e., this mode is a transverse-electric/longitudinal-magnetic one. Similarly only the longitudinal electric field component exists for the TM light mode, which is, thus, a transverse-magnetic/longitudinal-electric mode.
(3) We have $\operatorname{Re}\{\mathbf{S}\}=0$ for both TE and TM modes, which indicates that there is no power flow at all through these modes, i.e., these special surface modes are non-propagating or trapped modes.

\section{Some comments on the case of symmetric slab waveguides}

Finally we would like to comment on the mode characteristics of the symmetric slab waveguide made of (or double interfaces between) dual singly negative materials (e.g., $\mathcal{\varepsilon}$-negative-core and $\mu$-negative-clad structure). For this, we consider first a symmetric metalinsulator-metal (MIM) or insulator-metal-insulator (IMI) waveguide [see Fig. 2 (a) and (b)].

In this structure, the identical SPP modes (only for TM light) of the left and right interfaces ( $\psi_{l}$ and $\psi_{r}$

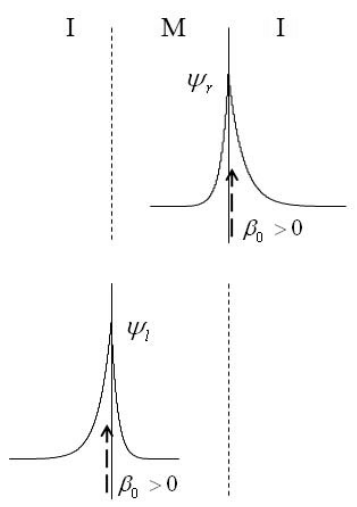

(a)
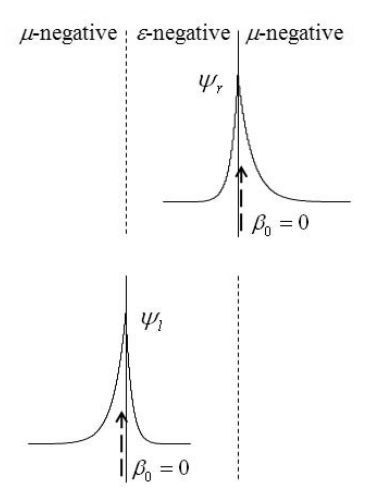

(c)

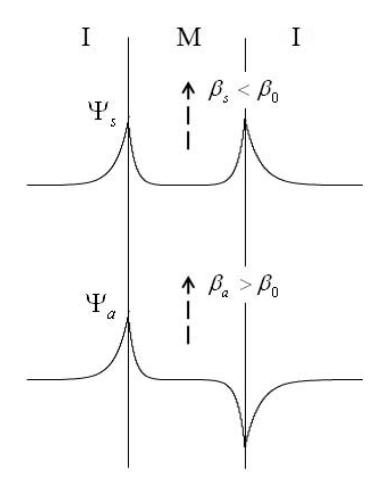

(b)

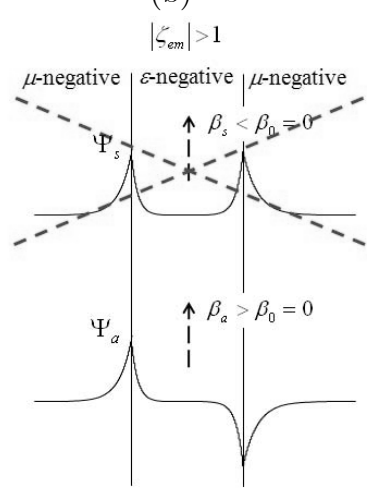

(d)
FIG. 2. (a) and (b) The case of IMI waveguide structure. Identical SPP modes of the left and right interfaces interact with each other and generate symmetric and anti-symmetric coupled supermodes having the propagation constants of $\beta_{s}$ and $\beta_{a}$ which satisfy $\beta_{s}<\beta_{0}<\beta_{a}$. (c) and (d) An example of the waveguide made of dual singly negative material ( $\mu$-negative/ $/$-negative/ $\mu$-negative) layers that supports only the anti-symmetric coupled supermode (when $\left|\zeta_{e m}\right|>1$ ). The interaction between left and right non-propagating surface modes cannot generate two supermodes and form only an anti-symmetric one because $\beta_{s}$ cannot become smaller than $\beta_{0}=0$. 
having the common propagation constant of $\beta_{0}$ ) interact with each other. This interaction results in (1) the formation of symmetric and anti-symmetric coupled supermodes $\left[\Psi_{s} \approx(1 / \sqrt{2})\left(\psi_{l}+\psi_{r}\right)\right.$ and $\left.\Psi_{a} \approx(1 / \sqrt{2})\left(\psi_{l}-\psi_{r}\right)\right]$ and (2) the splitting between the propagation constants of these two supermodes $\left(\beta_{s}\right.$ and $\left.\beta_{a}\right)$. The shorter the center slab is, the stronger the interaction between $\psi_{l}$ and $\psi_{r}$ becomes, resulting in the more prominent splitting between $\beta_{s}$ and $\beta_{a}$. It is notable that the symmetry here denotes that of the transversal field components $H_{y}$ and $E_{x}\left(\propto H_{y}\right)$, and the longitudinal field $E_{z}\left(\propto \partial H_{y} / \partial x\right)$ has the opposite symmetry. We have $\beta_{s}>$ $\beta_{0}>\beta_{a}$ in the MIM waveguide while $\beta_{a}>\beta_{0}>\beta_{s}$ in the IMI structure. This difference can be understood by considering the electric force acting on the electrons in metal layers. Let us consider the IMI structure first. The symmetric $E_{x}$ distribution in the waveguide makes the directions of $E_{x}$ at the left and right interfaces the same. This results in the anti-symmetric charge distributions at the left and right boundaries of the metal core and attractive force between them. This force moves the electrons toward the center of the core, which increases effectively the skin depth of the core metal, i.e., decreases the decay constant $\kappa_{m}$. From the relation $-\kappa_{m}^{2}+\beta^{2}=\varepsilon_{m} k_{0}^{2}$ at the metal having a dielectric constant $\mathcal{E}_{m}$, reduced $\kappa_{m}$ results in the decreased propagation constant and thus, we can get $\beta_{s}<\beta_{0}$. Similar consideration can show that the anti-symmetric $E_{x}$ causes repulsive force between the left and right boundaries of the metal core, reducing the skin depth. Thus, we have $\beta_{a}>\beta_{0}$. In the MIM waveguide, the symmetric $E_{x}$ also results in attractive force between the left and right interfaces. In this case, however, the force moves the electrons of the clad metal layers toward the core-clad interfaces, which reduces effectively the skin depth of the clad metal. Therefore, we have $\beta_{s}>\beta_{0}$ and similarly $\beta_{a}<\beta_{0}$.

However, in the slab waveguide made of dual singly negative materials, this kind of splitting is impossible because neither $\beta_{s}$ nor $\beta_{a}$ can be made smaller than $\beta_{0}=0$. Therefore, the interaction between left and right non-propagating surface modes generates only a single guiding mode. It is either a symmetric or an antisymmetric coupled one depending on the configuration of the waveguide structure and its propagation constant becomes always larger than $\beta_{0}=0$. For example, in the $\mu$-negative/ $\varepsilon$-negative/ $\mu$-negative structure, $\left|\zeta_{e m}\right|<1$ results in the anti-symmetric coupled supermode [see Fig. 2 (c) and (d)] while the symmetric coupled one exists when $\left|\zeta_{e m}\right|>1$ (mathematical details can be found in [4]).

\section{CONCLUSION}

In conclusion, we have shown that the single interface between dual singly negative media can support a special kind of surface mode, through which we can expect no power flow. This indicates that this mode is a non-propagating or trapped one. The corresponding light field becomes static along the longitudinal direction and thus, transverse-electric/longitudinal-magnetic or transverse-magnetic/longitudinal-electric modes are formed at the interface.

We can expect a few applications based on this non-propagating surface mode. We will briefly discuss two of them. As shown in [3], we can use the simple structure in Fig. 1 as a transmission filter under normal incidence (from left to right). By appropriately controlling the length of each singly negative layer, we can make only the normally incident light undergo total transmission with other light waves totally reflected. It is because total transmission occurs via mode couplings, from the incident to the transmitted plane waves intervened by the non-propagating surface mode, and only the normally incident light can satisfy the necessary phase matching condition with the mediate non-propagating mode. Another one is a waveguide-type total reflector. If we make a tapered slab waveguide and launch light fields where the slab width is small, the light waves propagate along the longitudinal direction with the slab width increasing. When the slab becomes so wide that the interaction between the left and right interfaces can be negligible, we can say that nonpropagating surface modes are formed near the left and right interfaces. In this case, the guided light waves cannot transmit through these non-propagating modes and get totally reflected since $\operatorname{Re}\{\mathbf{S}\}=0$ for all points along the transversal direction and the singly negative claddings do not allow so-called radiation or leaky modes. Therefore, we can implement the waveguidetype total reflector. Details on this will be discussed elsewhere.

\section{ACKNOWLEDGMENT}

This work was supported by the Korea Research Foundation Grant funded by the Korean Government (KRF-2008-331-D00399).

\section{REFERENCES}

1. S. A. Ramakrishna, "Physics of negative refractive index materials," Rep. Prog. Phys. 68, 449-521 (2005).

2. A. D. Boardman, N. King, and L. Velasco, "Negative refraction in perspective," Electromagnetics 25, 365-389 (2005).

3. K.-Y. Kim, J. Park, and B. Lee, "Complete tunneling of normally incident light through singly negative materials," Phys. Rev. A 79, 055801 (2009). 
4. K.-Y. Kim, I.-M. Lee, and B. Lee, "Guiding modes of a slab waveguide composed of impedance-matched single negative materials," IEEE Photon. Technol. Lett. 21, 736-738 (2009).

5. M. G. Silveirinha and N. Engheta, "Tunneling of electromagnetic energy through subwavelength channels and bends using $\mathcal{E}$-near-zero materials," Phys. Rev. Lett. 97,
157403 (2006).

6. I.-M. Lee, J. Jung, Y. Lim, and B. Lee, "Envelope-only pulse propagation in almost-dispersion-free matched metamaterials near frequency of zero refractive index," Jpn. J. Appl. Phys. 46, 5441-5446 (2007).

7. M. G. Silveirinha and N. Engheta, "Transporting an image through a subwavelength hole," Phys. Rev. Lett. 102, 103902 (2009). 